\title{
Nucleic Acid Therapeutics for Neurological Diseases
}

\author{
Jonathan K. Watts ${ }^{1}$ (D) Robert H. Brown $^{1,2}$ - Anastasia Khvorova ${ }^{1}$
}

Published online: 29 April 2019

(C) The American Society for Experimental NeuroTherapeutics, Inc. 2019

Oligonucleotide therapeutics are emerging as a promising class of therapeutics in various areas of human health. In the long run, oligonucleotides are likely to become a major class of therapeutics, on par with small molecules and biologics. Initially, oligonucleotides were predominantly used for modulation of gene expression in the liver, in which robust clinical efficacy has been demonstrated with both antisense $[1,2]$ and siRNA [3] compounds reaching approval.

Oligonucleotides do not cross the healthy blood-brain barrier. However, direct administration of oligonucleotides into the cerebrospinal fluid or the brain parenchyma supports reasonable distribution through the brain and spinal cord. The recent approval of nusinersen, a splice-switching antisense oligonucleotide (ASO) for the treatment of spinal muscular atrophy (SMA), has opened a new era in nucleic acid-based therapeutics for neurodegenerative diseases [4-6]. This first approval has now been followed by at least six additional antisense compounds in clinical trials [7] and several other platforms, such as siRNAs and AAV-expressed gene therapies, rapidly entering the field. This special issue of Neurotherapeutics provides insights into the current state of nucleic acid therapeutics for neurological diseases.

One of the most promising areas for oligonucleotide therapeutics is the treatment of genetically well-defined neurodegenerative disorders. For example, multiple drugs are in clinical trials for SOD1- and C9ORF72-driven amyotrophic

Jonathan K. Watts

jonathan.watts@umassmed.edu

Robert H. Brown

robert.brown@umassmed.edu

Anastasia Khvorova

anastasia.khvorova@umassmed.edu

1 RNA Therapeutics Institute, UMass Medical School, Worcester, Massachusetts 01605, USA

2 Department of Neurology, UMass Medical School, Worcester, Massachusetts 01605, USA lateral sclerosis (ALS) and Huntington's disease (HD), and MAPT (Tau)-driven Alzheimer's disease and frontotemporal dementia. In this issue of Neurotherapeutics, Zain and Smith [8] explore the potential for oligonucleotide therapeutics for the more than 30 repeat expansion disorders in the central nervous system (CNS), including HD, myotonic dystrophy, and Friedreich's ataxia. The authors consider the relative challenges and opportunities of DNA targeting (to stabilize tandem repeats) and RNA targeting (to modulate gene expression). Expanded repeats can also adopt unique structures which represent therapeutic opportunities [8].

Digging further into one type of repeat expansion disorder, the six spinocerebellar ataxias characterized by expansion of polyglutamine repeats, Buijsen et al. provide a detailed examination of progress in the genetics and treatment of this family of devastating disorders [9]. These diseases can be treated by targeting the polyglutamine repeat-containing gene itself as well as downstream genes that contribute to pathological mechanism(s).

The above diseases contain a single, clear, genetically validated target. Additional major classes of neurodegenerative disease, including Alzheimer's disease and Parkinson's disease (PD), are more complex multifactorial disorders. Multiple potential driver genes in combination with environmental factors and natural aging contribute to the disease progression. These complex landscapes create plentiful opportunities for possible therapeutic targets. As such, Nakamori et al. provide a review of current developments toward the treatment of $\mathrm{PD}$, in which multiple programs are rapidly advancing in preclinical development [10]. Interestingly, several targets relevant to PD (such as alpha-synuclein) are also relevant to multiple other neurological diseases, indicating potential convergence of neurodegenerative pathways.

The above areas of research have focused on chronic neurodegeneration. However, there are also exciting possibilities for nucleic acid therapeutics to make an impact in the treatment of acute neurological conditions. In this issue, Henninger and Mayasi review progress made on the use of nucleic acid therapies for the prevention, acute treatment, and 
recovery from ischemic stroke [11]. Nucleic acids may also constitute essential biomarkers to aid in stroke care: miRNA signatures may also enable classification of stroke subtypes for improving prevention of secondary strokes [11].

Neuroinflammation is a significant component of many aspects of neurodegeneration and is the primary driver of pathology in multiple sclerosis. Current strategies for nucleic acid therapeutics in neuroimmune disorders are reviewed by Greenfield and Hauser [12]. Thus, early and aggressive treatment of specific targets in specific cell populations using nucleic acid or cell therapies may slow the neurodegeneration that results from an aberrant immune function in these diseases.

All of the above topics have focused on the healthy or pathologically aging brain. There is also a significant future for the use of oligonucleotide therapeutics in the treatment of malignant brain disorders. As such, Krichevsky and Uhlmann provide a comprehensive overview of current and future advances in nucleic acid therapeutics for neuro-oncology [13]. Although much of the platform technology already developed for the healthy or aging brain may equally apply to brain tumors, brain cancers also provide unique challenges and opportunities. In terms of opportunities, early data suggest that it is significantly easier to deliver nucleic acid therapeutics through the disrupted blood-brain barrier at tumor sites. However, it is equally true that the favorable uptake of oligonucleotides demonstrated in healthy neurons and glia may not apply to all cells of all brain tumors. If delivery to brain tumors can be robustly achieved, the informational nature of nucleic acid therapeutics $[14,15]$, in which genetic information can intuitively and rapidly be used to define drug targets, lends itself well to the heterogeneous and dynamic genetics of brain tumors, and to the development of therapeutic approaches tailored to specific patients or even specific tumors. Thus, although the field is emerging, this approach has the potential to open new fundamental dimensions in the search for cures for gliomas and other brain tumors.

Finally, a very promising area of research in neurotherapeutics focuses not on the CNS but on peripheral sensory organs, which represent unique opportunities and challenges from a pharmacological perspective. As such, Hastings and Jones provide an update on ASO technology and discuss promising targets for inner ear-targeted therapeutics [16].

\section{Summary and Outlook}

The development of nucleic acid therapeutics for neurological diseases is rapidly evolving, with nusinersen approved and more than 10 nucleic acid drug candidates in clinical development for use in disorders of the CNS. Dozens more nucleic acid drugs for neurological targets are at the stage of early preclinical development.
There is a significant cross-talk in platform technology development for neurological diseases. A single AAV capsid that gives broad delivery to the brain may be used to deliver many cargo genes to treat different diseases. Similarly, if a chemical modification pattern is functional and nontoxic for one antisense oligonucleotide, it is an ideal starting point for the development of others (though of course there is still some selection and optimization required). We have recently coined the term "dianophore" to describe the ensemble of molecular features that determine the distribution and pharmacokinetic parameters of a drug. This term is contrasted with the pharmacophore, which is the ensemble of molecular features that determine target recognition and modulation [15].

By way of illustration, it took many years of focused work for Ionis and Biogen to obtain the first approval of nusinersen as an antisense oligonucleotide for use in the CNS. But using the same chemical modification pattern as was used in nusinersen, the group of Timothy Yu (Harvard Medical School) was able to develop a custom drug for a single pediatric patient with Batten disease in a matter of months [17]. Thus, we expect that the pace of development of nucleic acid therapeutics for neurological diseases will continue to accelerate in the coming years.

There is also a significant cross-talk in terms of gene targets between different neurological conditions. Although the initial trigger of neurodegeneration is different in each disease, in many cases, downstream pathways of neurodegeneration and inflammation are shared and, thus, one target may be applicable to the treatment of multiple genetically distinct diseases. The most advanced nucleic acid drug candidates are focused on well-defined monogenic diseases such as spinal muscular atrophy, ALS, and HD. But a major advantage of nucleic acid therapeutics is the ability to simultaneously address multiple targets because oligonucleotides of many sequences can have similar pharmacokinetic properties. This is likely to be particularly important for the treatment of complex neurodegenerative disorders including $\mathrm{AD}$ and $\mathrm{PD}$. It is even more likely to be required for therapeutics in the fields of neuro-oncology and trauma.

Required Author Forms Disclosure forms provided by the authors are available with the online version of this article.

\section{References}

1. Raal, F.J., Santos, R.D., Blom, D.J. et al. (2010). Mipomersen, an apolipoprotein B synthesis inhibitor, for lowering of LDL cholesterol concentrations in patients with homozygous familial hypercholesterolaemia: a randomised, double-blind, placebo-controlled trial. Lancet, 375, 998-1006.

2. Benson, M.D., Waddington-Cruz, M., Berk, J.L. et al. (2018). Inotersen treatment for patients with hereditary transthyretin amyloidosis. N Engl J Med, 379, 22-31. 
3. Adams, D., Gonzalez-Duarte, A., O'Riordan, W.D. et al. (2018). Patisiran, an RNAi therapeutic, for hereditary transthyretin amyloidosis. N Engl J Med, 379, 11-21.

4. Chiriboga, C.A., Swoboda, K.J., Darras, B.T. et al. (2016). Results from a phase 1 study of nusinersen (ISIS-SMN(Rx)) in children with spinal muscular atrophy. Neurology, 86, 890-897.

5. Aartsma-Rus, A. (2017). FDA Approval of nusinersen for spinal muscular atrophy makes 2016 the year of splice modulating oligonucleotides. Nucleic Acid Ther, 27, 67-69.

6. Corey, D.R. (2017). Nusinersen, an antisense oligonucleotide drug for spinal muscular atrophy. Nat Neurosci, 20, 497-499.

7. Seguin, R. in Oligonucleotide-Based Drugs and Therapeutics: Preclinical and Clinical Considerations for Development. (eds. N. Ferrari \& R. Seguin) pp. 389-409 (Wiley, Hoboken; 2018).

8. Zain R, Smith CIE. Targeted oligonucleotides for treating neurodegenerative tandem repeat diseases. Neurotherapeutics 2019; this issue.

9. Buijsen RAM, Toonen LJA, Gardiner SL, van Roon-Mom WMC. Genetics, mechanisms and therapeutic progress in polyglutamine spinocerebellar ataxias. Neurotherapeutics 2019; this issue.

10. Nakamori M, Junn E, Mochizuki H, Mouradian MM. Nucleic acid based therapeutics for Parkinson's disease. Neurotherapeutics 2019; this issue.
11. Henninger N, Mayasi Y. Nucleic acid therapies for ischemic stroke. Neurotherapeutics 2019; this issue.

12. Greenfield AL, Hauser SL. Nucleic acid-based therapeutics relevant to neuroimmune conditions. Neurotherapeutics 2019; this issue.

13. Krichevsky A, Uhlmann EJ. Oligonucleotide therapeutics as a new class of drugs for malignant brain tumors: targeting mRNAs, regulatory RNAs, mutations, combinations, and beyond. Neurotherapeutics 2019; this issue.

14. Cohen, J.S. (1991). Informational drugs: a new concept in pharmacology. Antisense Res Dev, 1, 191-193.

15. Khvorova, A. \& Watts, J.K. (2017). The chemical evolution of oligonucleotide therapies of clinical utility. Nat Biotechnol, 35, 238-248.

16. Hastings ML, Jones TA. Antisense oligonucleotides for the treatment of inner ear dysfunction. Neurotherapeutics 2019; this issue.

17. Kaiser, J. (2018). A tailormade drug developed in record time may save girl from fatal brain disease. Science. https://doi.org/10.1126/ science.aav7907.

Publisher's Note Springer Nature remains neutral with regard to jurisdictional claims in published maps and institutional affiliations. 